\title{
Harmful Interference of Detoxifying Diets and Nutraceuticals with Adherence to Abemaciclib in Advanced Estrogen Receptor-Positive, Human Epidermal Growth Factor Receptor-2- Negative Breast Cancer: A Case Report
}

\author{
Vittorio Gebbia ${ }^{a}$ b Dario Piazza $^{c}$ Maria Rosaria Valerio ${ }^{d}$ \\ aMedical Oncology Unit, La Maddalena Clinic for Cancer, Palermo, Italy; ${ }^{b}$ Department \\ of Health Promotion, Mother and Child Care, Internal Medicine and Medical Specialties \\ ProMISE, University of Palermo, Palermo, Italy; ' $\mathrm{C} S T$ T Foundation, Palermo, Italy; ${ }^{\mathrm{d}}$ Medical \\ Oncology Unit, Policlinic Paolo Giaccone, University of Palermo, Palermo, Italy
}

Keywords

Metastatic breast cancer - Estrogen receptor · Abemaciclib - Detoxifying diet · Nutraceuticals

\begin{abstract}
Many cancer patients use integrative therapies with a combination of natural products and diets. In the Western world, integrative medicine is often not shared with oncologists even during antineoplastic treatments. This behavior stems from the unmet needs of cancer patients who may feel oncologists' underestimation of their symptoms and spiritual aspects. This case report demonstrates the potential harm of inadequate diet and nutraceutical intake in a 68-year-old woman with metastatic estrogen receptor-positive, human epidermal growth factor receptor2-negative breast cancer. Her care team recommended hormone therapy with abemaciclib plus fulvestrant. Her diarrhea started after 10 days of therapy and did not disappear, despite the use of loperamide, causing a significant reduction in adherence and dose intensity of abemaciclib. The patient finally disclosed to her oncologist she was following a detoxifying diet and taking several nutraceuticals. Her diarrhea was correlated with abemaciclib but most probably exacerbated and prolonged by the diet. Evaluation of disease after 3 months showed progressive disease. Integrative medicine should be in the multidisciplinary management of cancer patients to avoid potentially harmful events and ameliorate patients' quality of life in a holistic approach.
\end{abstract}




\section{Introduction}

Metastatic breast cancer still represents a major cause of death in women [1]. To date, patients with estrogen receptor-positive, human epidermal growth factor receptor-2-negative (her-2) breast carcinoma are best managed with a combination of antihormonal agents and cyclin-dependent kinase 4 and 6 inhibitors. Among the latter class, abemaciclib is the most potent in preclinical studies [2]. Such combination therapy may achieve a median disease-free survival of 46 months with good tolerability in breast cancer patients progressing during endocrine therapy [3].

Recently, the role of integrative medicine has gained popularity in a more open, multidisciplinary, holistic way of interpreting oncology and unmet patients' needs [4-6]. Many patients follow detoxifying or anticancer diets and take nutraceuticals often without discussion with their treating oncologists [7]. This attitude toward the use of nutraceuticals should be carefully evaluated in a team of integrative medicine since natural products does not always correspond to safety in oncology [8]. This article reports a negative interaction between detoxifying diet and nutraceuticals with adherence to antihormonal therapy in a woman with advanced estrogen receptor-positive, her-2-negative breast cancer.

\section{Case Report}

Written informed consent was obtained from the patient for publication of this case report and any accompanying images. A 68-year-old woman - a housewife - presented with suspect progressive breast cancer while taking adjuvant letrozole for 3 years. In February 2018, she was diagnosed with breast cancer and received conservative left breast surgery with axillary dissection after sentinel lymph node analysis was positive for cancer. The systemic staging was negative for metastatic disease. Pathology showed a ductal infiltrating carcinoma estrogen receptor $80 \%$, progestin receptor 35\%, her-2 score 1, and Ki67 40\%. The clinical and pathological stage was pT2, N1, M0. She received an adjuvant chemotherapy regimen with epirubicin and cyclophosphamide every 3 weeks for 4 cycles, followed by paclitaxel for 12 weeks, complementary radiotherapy on the left breast and the homolateral axilla, and started adjuvant letrozole. In May 2020, she was admitted to our outpatients' clinic because of a Ca15.3 increase. A positron emission tomography/CT (PET/CT) showed progressive disease at nodes, bone, and an unspecific liver uptake. Physical examination was nonsignificant, and the patient denied any symptoms of the disease but moderate fatigue. She was classified as an estrogen receptor-positive, her-2/neu-negative, hormone-resistant breast carcinoma.

The oncologist quitted letrozole. Based on scientific data, the oncologist proposed a systemic treatment with fulvestrant $500 \mathrm{mg}$ every 2 weeks as a loading dose 3 times and then every 4 weeks, plus abemaciclib $150 \mathrm{mg}$ bid on a continuous schedule [3]. The patient's performance status was adequate for abemaciclib plus fulvestrant regimen with an Eastern Cooperative Oncology Group Performance Status score of 1. The oncologist carefully interviewed the patient for concomitant gastrointestinal diseases that could contraindicate abemaciclib use and other drug assumptions. The oncologist performed a drug-drug interaction evaluation employing a drug checker tool and explained in detail abemaciclib to the patient and her daughter, including written precise suggestions concerning potential side effects. More specifically, the oncologist stressed the precocious use of loperamide and dietary modification in case of diarrhea. He recommended immediately taking loperamide at first or second liquid stool and contacting the medical oncology team via a WhatsApp messenger system (Fig. 1).

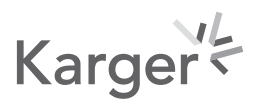




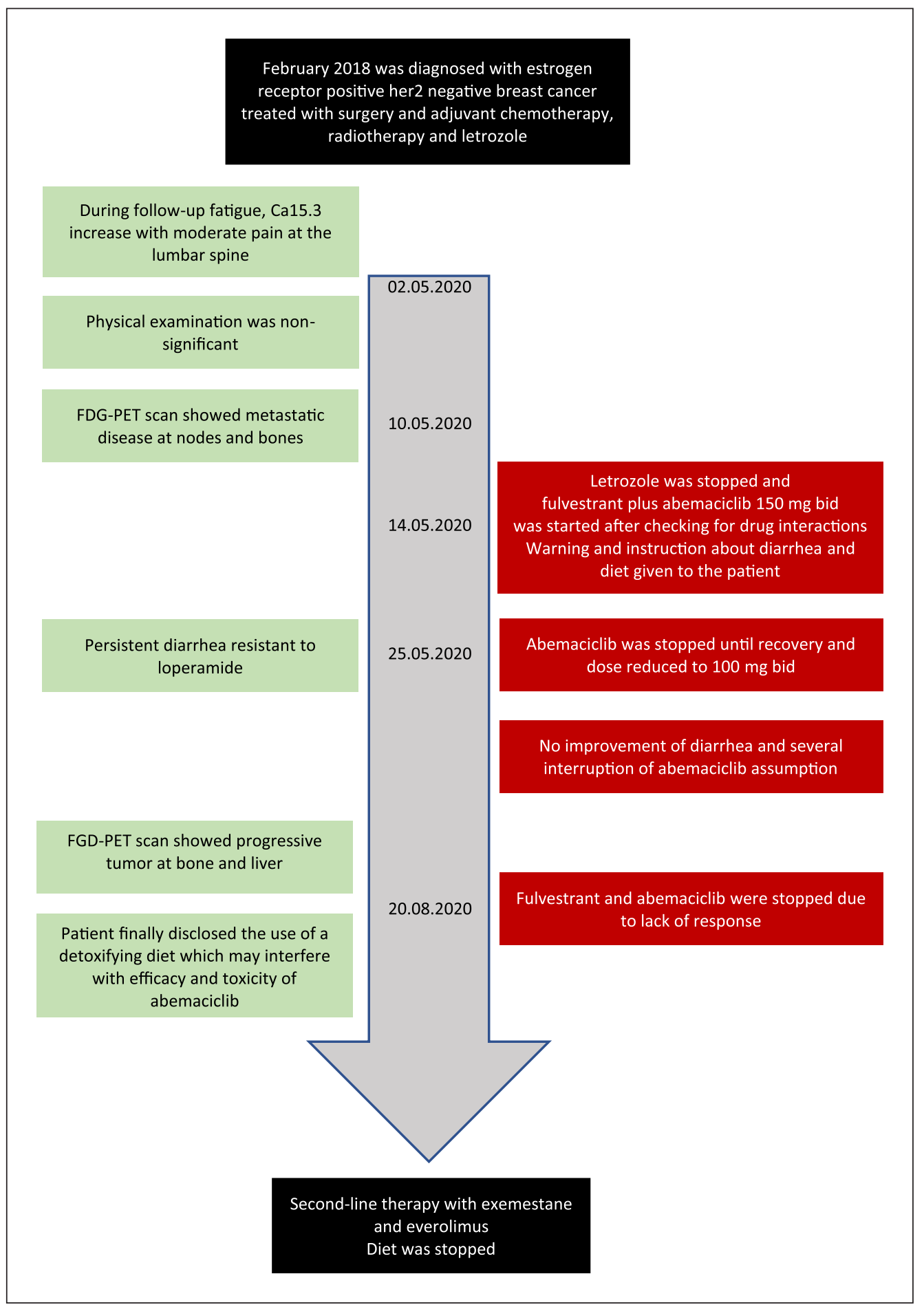

Fig. 1. Treatment timeline.

After 10 days, she complained of grade 2 diarrhea, according to the National Cancer Institute Common Toxicity Criteria version 5.0 [9]. Nurses called the patient to assure she was following a correct astringent diet and loperamide assumption. A blood test showed only a mild increase in serum creatinine. Since she reported intermittent diarrhea, the oncologist 
reduced the abemaciclib dose to $100 \mathrm{mg}$ bid, but intestinal movements remained unchanged and stopped abemaciclib for 3 days. Such drug-free periods occurred every 2 weeks, with no other significant side effects.

At this point, the patient disclosed she was following a detoxifying diet prescribed by a dietician. Oncologists were not aware of it. The dietician did not make any effort to contact the managing oncologists. Table 1 depicts the diet components. As shown in Table 2, she was also taking several integrative agents such as broccoli extracts ( $250 \mathrm{mg}$ bid), garlic extracts (500 mg bid), high-dose vitamin D, curcumin plus black pepper (526 mg bid), green tea (3 serving/day), vitamin C (500 mg/day), lipoic acid $100 \mathrm{mg} /$ day, coenzyme q10 $100 \mathrm{mg} /$ day, vitamin $\mathrm{K}$ (250 mcg/day), selenium $(75 \mathrm{mcg} /$ day $)$, and iodine $(150 \mathrm{mcg} /$ day $)$. The dietary prescription also reported that "people may experience weakness, stool modification including diarrhea, and generalized pain, which are positive signs of detoxification." Accordingly, the patient showed evident difficulties in decoding her gastrointestinal symptoms. The oncologist stopped the diet, and diarrhea frequency reduced in 2 weeks. The dose intensity of abemaciclib was $68.5 \%$ of the planned one, according to the Hyrnuk and Bush formula [10]. After 3 months of abemaciclib and fulvestrant, a new PET/CT showed progressive disease according to the RECIST criteria. Besides reversible diarrhea G3 and renal toxicity (creatinine) G1, no other significant adverse events were observed according to the NCCN-CTC criteria [9]. The oncologist's decision was to withheld fulvestrant and abemaciclib and proposed a second-line treatment of everolimus and exemestane. She is still alive during the time of writing.

\section{Discussion}

Abemaciclib may be administered with or without food, and food-drug interaction analysis showed that diet has modest effects on the pharmacokinetics of abemaciclib [11]. A high-fat, high-calorie meal administered to healthy subjects increased the C-max and AUC of abemaciclib plus its active metabolites by $26 \%$ and $9 \%$, respectively. Grapefruit juice may increase the plasma concentrations of abemaciclib and therefore should be avoided. The possible mechanism is the inhibition of CYP450 3A4-mediated first-pass metabolism in the gut wall by certain compounds present in grapefruit. Inhibition of hepatic CYP450 3A4 may also contribute. According to product labeling, abemaciclib systemic exposure (AUC) is predicted to increase up to 16 -fold when administered with the potent CYP450 3A4 inhibitor ketoconazole.

In this study, we report the inappropriate use of a detoxifying diet with a massive daily administration of herbal and nutraceutical agents in a female patient treated with fulvestrant plus abemaciclib for metastatic breast cancer. This patient showed poor response to antihormonal therapy, as shown by PET/CT, and reported chronic diarrhea, which hampered treatment adherence and drug dose intensity by one-third of the planned dose. Neither the patient nor the family caregiver or the dietician informed the oncology center. Suspension of the diet by the treating oncologist resulted in the weakening of diarrhea. Although usual diet habits seem not to influence abemaciclib pharmacokinetics, an incorrect diet may cause gastrointestinal disturbances. These may favor the occurrence of abemaciclib-related diarrhea, causing dose reductions and poor adherence and eventually negatively influencing patients' outcomes and quality of life.

Our patient was taking many compounds with possible negative interaction on treatment outcome. Supplementation with coenzyme q10 and vitamins caused a more unsatisfactory outcome in patients with metastatic breast cancer treated with chemotherapy in a Southwest Oncology Group trial [12]. Although the herb-drug interaction risk for short-term use of garlic

\section{Karger'}




\section{Case Reports in Oncology}

Table 1. Diet composition

Gebbia et al.: Interference of Detoxifying Diets

Breakfast, $g$

Almond milk

Coconut yogurt

200

Puffed cereals

30

Midmorning snack, g

Carrots

Avocado

40

Olives

Dried fruit

Lunch, g

Cooked greens

Quinoa

80

Cooked legumes

Olive oil

40

Oily dried fruit

Afternoon snack, g

Avocado

80

Olives

Oily dried fruit

20

Fresh coconut

Coconut yogurt

Dinner, g

Fish

150

Egg

120 (2 eggs)

Cooked vegetables

200

Olive oil

40

Banned aliments

Lemon

Cheese

Chocolate

Tomato

Eggplant

Maize

Potato

Peppers

Cooked carrots

Alcohol drinks

Dried figs

Dried plums

Peanuts 


\section{Case Reports in Oncology}

Table 2. Nutraceuticals

\begin{tabular}{l}
\hline Case Rep Oncol 2021;14:1399-1406 \\
\hline DOI: 10.1159/000518779
\end{tabular} \begin{tabular}{l}
$\begin{array}{l}\odot \text { 2021 The Author(s). Published by S. Karger AG, Basel } \\
\text { www.karger.com/cro }\end{array}$ \\
\hline
\end{tabular}

Gebbia et al.: Interference of Detoxifying Diets

\begin{tabular}{ll}
\hline Agent & Dosage \\
\hline Broccoli extracts & $250 \mathrm{mg}$ bid \\
Garlic extracts & $500 \mathrm{mg}$ bid \\
Curcumin & $526 \mathrm{mg}$ bid \\
Coenzyme q10 & $100 \mathrm{mg} /$ once a day \\
Vitamin K & $250 \mathrm{mcg} /$ once a day \\
Vitamin C & $500 \mathrm{mg} /$ once a day \\
Lipoic acid & $100 \mathrm{mg} /$ once a day \\
Selenium & $75 \mathrm{mcg} /$ once a day \\
Iodine & $150 \mathrm{mcg} /$ once a day \\
Green tea & Three serving/day \\
\hline
\end{tabular}

is low, prolonged exposure to concentrated garlic extracts may counteract the efficacy of drugs whose disposition depends on the human efflux transporter ABCB1 which may occur in tumor cells $[13,14]$. Even if the check for interactions between the single nutraceuticals and antihormonal agents employed in our patient was negative, knowledge on the possible effects of the whole "orchestra" of agents is poor.

In clinical practice, oncologists commonly check drug-drug interactions when prescribing modern targeted therapies. On the other hand, herb-drug or food/diet-drug is far less explored, even if herb-drug interaction checkers are available [15]. In this patient, while such a check did not show negative interaction between antihormonal agents and supplements, the diet followed caused a sharpening and prolongation of liquid stools, causing poor adherence and dose reduction of abemaciclib. Moreover, the reckless pieces of information about possible effects of diet, such as fatigue and loose stools, elicited confusion in the patient. Today, herbal supplements and nutraceuticals in various forms can be easily purchased over the counter and widely employed by cancer patients, often without discussing with their oncologists. This hidden behavior is probably related to sometimes justified patients' perception that their needs in terms of quality of life are underweighted by most oncologists far more concentrated on therapy management [16]. Herbal supplements and nutraceuticals are often self-prescribed or suggested by friends, other patients, or prescribed by professionals other than oncologists. Such prescriptions are often not shared, even if patients should discuss its use with the managing oncologist and pharmacist. The regulatory agencies do not pay the same attention for pharmaceutical drugs to herbals, dietary supplements, and their manufacturers.

\section{Conclusion}

Although integrative medicine has a significant positive role in managing people affected by cancer, a careful approach is advisable since herbal supplements and nutraceuticals may enhance prescription medications' side effects and block the intended therapeutic drug efficacy. It is then advisable that deep and correct information be delivered to patients about the potential benefits but especially about the possible interactions of herbal supplements and nutraceuticals that may occur with the standard therapies administered. For this purpose, a multidisciplinary approach is essential to treat this kind of patients.

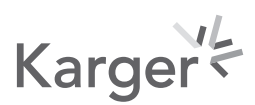




\section{Case Reports in Oncology}

\begin{tabular}{l|l}
\hline Case Rep Oncol 2021;14:1399-1406 \\
\hline DOI: 10.1159/000518779 & $\begin{array}{l}\text { ○ 2021 The Author(s). Published by S. Karger AG, Basel } \\
\text { www.karger.com/cro }\end{array}$ \\
\hline
\end{tabular}

Gebbia et al.: Interference of Detoxifying Diets

\section{Acknowledgment}

This study was conducted at the La Maddalena Cancer Center, Palermo, Italy.

\section{Statement of Ethics}

The study is exempt from ethics committee approval because every diagnostic and therapeutic action for the primary pathology was performed according to the current standards and guidelines. Written informed consent was obtained from the patient for publication of this case report and any accompanying images.

\section{Conflict of Interest Statement}

The authors declared no potential conflicts of interest concerning the research, authorship, and/or publication of this article.

\section{Funding Sources}

The authors disclosed receipt of the following financial support for the research, authorship, and/or publication of this article: this study was supported by the Network in Integrative Medicine in Oncology Program of the GSTU Foundation, Palermo, Italy.

\section{Author Contributions}

V.G. and M.R.V. contributed to study concept and design. V.G. and D.P. contributed to drafting of the manuscript. All authors contributed to acquisition, analysis, and interpretation of data, critical revision of the manuscript, and read and approved the final manuscript.

\section{Data Availability Statement}

All data generated or analyzed during this study are included in this article. Further inquiries can be directed to the corresponding author.

\section{References}

1 Bray F, Ferlay J, Soerjomataram I, Siegel RL, Torre LA, Jemal A. Global cancer statistics 2018: GLOBOCAN estimates of incidence and mortality worldwide for 36 cancers in 185 countries. CA Cancer J Clin. 2018;68(6):394-424.

2 Gebbia V, Valerio MR, Firenze A, Vigneri P. Abemaciclib: safety and effectiveness of a unique cyclin-dependent kinase inhibitor. Expert Opin Drug Saf. 2020 Aug;19(8):945-54.

3 Sledge GW, Toi M, Neven P, Sohn J, Inoue K, Pivot X, et al. The effect of abemaciclib plus fulvestrant on overall survival in hormone receptor-positive, ERBB2-negative breast cancer that progressed on endocrine therapyMONARCH 2: a randomized clinical trial. JAMA Oncol. 2020 Jan 1;6(1):116-24.

4 Seely DM, Weeks LC, Young S. A systematic review of integrative oncology programs. Curr Oncol. 2012 Dec; 19(6):e436-61.

5 Chiesi F, Bonacchi A, Primi C, Miccinesi G. Assessing unmet needs in patients with cancer: an investigation of differential item functioning of the needs evaluation questionnaire across gender, age and phase of the disease. PloS One. 2017;12(7):e0179765. 
6 Ben-Arye E, Portalupi E, Keshet Y, Bonucci M, Can G, Kading Y, et al. Enhancing palliative care with mindful touch: impact of a manual and movement therapy training program in an international multidisciplinary integrative oncology setting. J Pain Sympt Manag. 2021 Aug;61(2):229.

7 Berretta M, Della Pepa C, Tralongo P, Fulvi A, Martellotta F, Lleshi A, et al. Use of complementary and alternative medicine (CAM) in cancer patients: an Italian multicenter survey. Oncotarget. 2017 Apr;8(15):2440114.

8 Schiff E, Levy I, Arnon Z, Ben-Arye E, Attias S. First, keep it safe: integration of a complementary medicine service within a hospital. Int J Clin Pract. 2018 May;72(5):e13082.

9 U.S. Department of Health and Human Services. Common Terminology Criteria for Adverse Events (CTCAE) [Internet]. Cancer.gov; 2017 [cited 2021 Jun 11]. Available from: https://ctep.cancer.gov/protocoldevelopment/electronic_applications/docs/CTCAE_v5_Quick_Reference_8.5x11.pdf.

10 Hryniuk W, Bush $\mathrm{H}$. The importance of dose intensity in chemotherapy of metastatic breast cancer. J Clin Oncol. 1984 Nov;2(11):1281-8.

11 Verzenios-epar-product-information_en.pdf [Internet]. [cited 2020 Nov 11]. Available from: https://www. ema.europa.eu/en/documents/product-information/verzenios-epar-product-information_en.pdf.

12 Ambrosone CB, Zirpoli GR, Hutson AD, McCann WE, McCann SE, Barlow WE, et al. Dietary supplement use during chemotherapy and survival outcomes of patients with breast cancer enrolled in a cooperative group clinical trial (SWOG S0221). J Clin Oncol. 2020 10;38(8):804-14.

13 Bayan L, Koulivand PH, Gorji A. Garlic: a review of potential therapeutic effects. Avicenna J Phytomed. 2014 Jan; 4(1):1-14.

14 Choi KH, Chen CJ, Kriegler M, Roninson IB. An altered pattern of cross-resistance in multidrug-resistant human cells results from spontaneous mutations in the mdr1 (P-glycoprotein) gene. Cell. 1988 May;53(4):519-29.

15 Drug-nutrient interactions [Internet]. Available from: https://www.integrativepro.com/Resources/DrugNutrient-Interaction-Checker [cited 2020 Nov 11].

16 van der Sluijs C, Lombardo FL, Lesi G, Bensoussan A, Cardini F. Social and Cultural Factors Affecting Complementary and Alternative Medicine (CAM) use during menopause in Sydney and Bologna. Evid Based Complement Alternat Med. 2013;2013:836234. 